\title{
Coordinating Storage and Demand Response for Microgrid Emergency Operation
}

\author{
C. Gouveia, J. Moreira, C. L. Moreira, J. A. Peças Lopes, Senior Member, IEEE
}

\begin{abstract}
Microgrids are assumed to be established at the low voltage distribution level, where distributed energy sources, storage devices, controllable loads and electric vehicles are integrated in the system and need to be properly managed. The microgrid system is a flexible cell that can be operated connected to the main power network or autonomously, in a controlled and coordinated way. The use of storage devices in microgrids is related to the provision of some form of energy buffering during autonomous operating conditions, in order to balance load and generation. However, frequency variations and limited storage capacity might compromise microgrid autonomous operation. In order to improve microgrid resilience in the moments subsequent to islanding, this paper presents innovative functionalities to run online, which are able to manage microgrid storage considering the integration of electric vehicles and load responsiveness. The effectiveness of the proposed algorithms is validated through extensive numerical simulations.
\end{abstract}

Index Terms-- Demand Response, Electrical Vehicles, Energy Storage, Islanding Operation, MicroGrid, Vehicle-to-Grid.

\section{NOMENCLATURE}

EV - Electric Vehicles

LC - Load Controller

LV - Low Voltage

MC - Microsource Controller

MG - Microgrid

MGCC - Microgrid Central Controller

MS - Microsource

MV - Medium Voltage

SOC - State of Charge

SOFC - Solid Oxide Fuel Cell

SSMT - Single Shaft Microturbine

VC - Vehicle Controller

\section{I.INTRODUCTION}

$\mathrm{M}$ eeting high reliability requirements in distribution systems with increasing penetration levels of distributed energy resources and future massification of Plugged-in

This work is funded by the ERDF - European Regional Development Fund through the COMPETE Program (operational program for competitiveness) and by National Funds through the FCT - Fundação para a Ciência e a Tecnologia (Portuguese Foundation for Science and Technology) within project Microgrids+EV: Identification of Control and Management Strategies for Microgrids with Plugged-in Electric Vehicles, PTDC/EEAEEL/103546/2008-(FCOMP-01-0124-FEDER-009866).

C. Gouveia, J. Moreira, C. L. Moreira and J. P. Lopes are with the INESC TEC-INESC Technology and Science (formerly INESC Porto), Rua Dr. Roberto Frias, 378, 4200 - 465, Porto, Portugal (e-mail: cstg@inescporto.pt, ee07250@fe.up.pt, carlos.moreira@inescporto.pt, jpl@fe.up.pt).
Electric Vehicles (EV) is a challenging task [1], [2]. In order to ensure the security and quality of supply, innovative decentralized control architectures should be developed at the distribution level, aiming a higher system observability and controllability, while assuring better control and management performances. The materialization of this scenario is in line with the Smart Grid development, in which the MicroGrid (MG) plays a central role [2].

The MG has been defined as a flexible cell of the Low Voltage (LV) distribution system, which incorporates local generation, based on renewable energy sources or low carbon technologies for combined heat and power applications, storage devices, responsive loads [3], [4] and in the future the $\mathrm{EV}$, which can behave both as flexible loads and mobile energy storage devices [5], [6].

The MG management and control system extends and decentralizes the distribution network monitoring and control capability, providing the adequate framework to fully integrate smart grid new players, namely the EV and the consumers (that can also become electric power producer - prosumers when installing microgeneration units) [2], [5], [6].

Being an extremely flexible cell of the electrical power system, the MG is able to operate interconnected to the Medium Voltage (MV) network (normal operating mode) or autonomously (emergency operating mode), if endowed with proper control and management system, in order to coordinate local generation resources, storage devices and flexible loads [7]. However, ensuring a successful MG autonomous operation is quite challenging. When operating autonomously, the MG stability relies in voltage and frequency control strategies, implemented locally through a network of local controllers.

Sudden islanding of the MG may cause high unbalances between local load and generation. Due to the slow response of Microsources (MS) to control signals, the power balance needs to be ensured by fast acting storage units, such as flywheels, complemented by load shedding schemes. Flywheels are able to inject/absorb significant energy during a short period of time (a $25 \mathrm{kWh}$ flywheel is able to provide 100 $\mathrm{kW}$ of power during $15 \mathrm{~min}$ ), requiring the adoption of specific storage management strategies in order to ensure the MG survival during autonomous operation [7].

The development of smart charging strategies exploiting the control flexibility of EV connected to the LV network, enable their active participation in the $\mathrm{MG}$ regulation strategies [5], [6]. However, global contribution from EV depends on the number of units connected to the system and 
on its batteries state of charge.

In addition to the control flexibility provided by $\mathrm{EV}$, the development of innovative demand response strategies dedicated to $\mathrm{LV}$ consumers may contribute to improve $\mathrm{MG}$ stability, particularly during emergency conditions. During islanded operation the major concern relies in the MG primary and secondary frequency regulation. Therefore, the most interesting demand response services are related to the provision of primary and secondary reserve. This type of service requires fast responses to transients, being usually implemented based on the system frequency. In [8] the authors proposed the FAPER concept - Frequency Adaptive Power Energy Reschedule - which switches on/off one or a group of equipment, based on the local frequency measurement. More recently, a load reduction algorithm was proposed in [9], considering the local frequency measurement from smart meters. The load control method aggregates typical domestic appliances, according to the maximum admissible time which the loads can be disconnected without harming consumers comfort. Thermostat controlled loads and appliances with long running cycles will disconnect for smaller frequency disturbances $(>0.3 \mathrm{~Hz})$ and during longer periods of time $(5$ min), while resistive and lighting loads will only be disconnected for frequency disturbances higher than $0.7 \mathrm{~Hz}$. An algorithm integrated in the smart meter determines the switching on and off of each type of equipment, sending the corresponding control signal to the loads controllers. The load control method developed was tested considering a single bus model of the Great Britain power system, showing improvements on the system stability. However, the authors also concluded that in order to provide primary frequency regulation, the system requires fast frequency measurements (with a maximum 1s delay). In [10], the authors developed a Dynamic Demand Control method dedicated in particular to the on/off control of refrigerators. The authors propose an alternative controller to the conventional thermostat, which will connect/disconnect the refrigerator based on the frequency in addition to the freezer air temperature. This method avoids additional disturbances due to the on/off cycles of conventional thermostat controlled refrigerators. The obtained results have shown that the proposed method potentially improves power system stability when facing the sudden loss of generation, increase of load, or when dealing with a large penetration of variable generation.

Regarding the MG operation, a central demand response strategy for the MG primary frequency regulation was proposed in [11]. The method is divided in three modes, defined according to the MG frequency behavior following a disturbance. Mode 0 corresponds to normal operating mode where no control is required. However, when the frequency deviation surpasses a pre-defined limit, the algorithm activates mode 1 and controls the load in order to bring the frequency within admissible range. The amount of load to be reconnected/disconnected is determined based on the adaptive hill climbing technique, which determines the minimum percentage of responsive loads which should be disconnected or connected based on the MG frequency deviation. The commands will then be sent by the central controller to the electric water heaters controllers. When the frequency returns to the admissible range of operation, mode 2 is activated, sending reconnection/disconnection orders to the loads participating in mode 1 .

Contrarily to the frequency responsive load control methods described in [9] and [10], the MG central demand response scheme proposed in [11] requires a robust communication network between the central controller and the load controllers, which should ensure a maximum latency of $500 \mathrm{~ms}$. When considering load control methods based on local frequency measurements, only an accurate local frequency measurement is required. The method proposed in [11] was tested for a distribution system integrating a synchronous machine. However, in the absence of synchronous machines, the MG frequency response will depend on the storage unit and controllable MS response to control signals. In order to ensure the MG stability during islanded operation, the $\mathrm{MG}$ requires sufficient storage capacity to ensure first local power balance [7], [12].

Taking into consideration the contribution of load control for the MG primary frequency regulation, this paper proposes an emergency demand response scheme for MG autonomous operation based on local frequency measurements. The active participation of MG loads will contribute to ensure the stability of the MG in the moments subsequent to islanding, taking into account frequency behavior and available energy in storage units. The proposed control strategy is supported by an online tool integrated into the MG central controller, which is responsible for periodically define the most adequate technical solution for managing responsive loads (including EV), following an unplanned event and taking into account the MG operating conditions. The proposed methodology is intended to manage demand response in the MG in short term periods following islanding. Other approaches are required to manage MG load responsiveness for islanding operation during larger time frames.

\section{II.OVERVIEW OF MG MANAGEMENT AND CONTROL}

The MG management and control system is assumed to be organized under a hierarchical structure. As shown in Fig. 1, the system is organized in a network of local controllers headed by the MG Central Controller (MGCC). The MGCC is installed at the MV/LV substation and concentrates the high level decision making for the technical management of the MG, being responsible for coordinating all the MG resources. The MG local controllers will periodically send to the MGCC information related with power consumption, generation, voltage and other data. The MGCC will then filter and process the information in order to manage the MG operation.

Considering the different $\mathrm{MG}$ possible actors, there are three types of local controllers: the Load Controller (LC), the Microsource Controller (MC) and the EV Controller (VC), acting as interfaces to locally control loads, EV charging and MS active and reactive power production levels [5], [6]. The control of the MG elements is based on local measurements, avoiding the dependence on fast communication [7]. However, 
supervisory control is always necessary in order to adequately manage the available resources in a larger time frame.

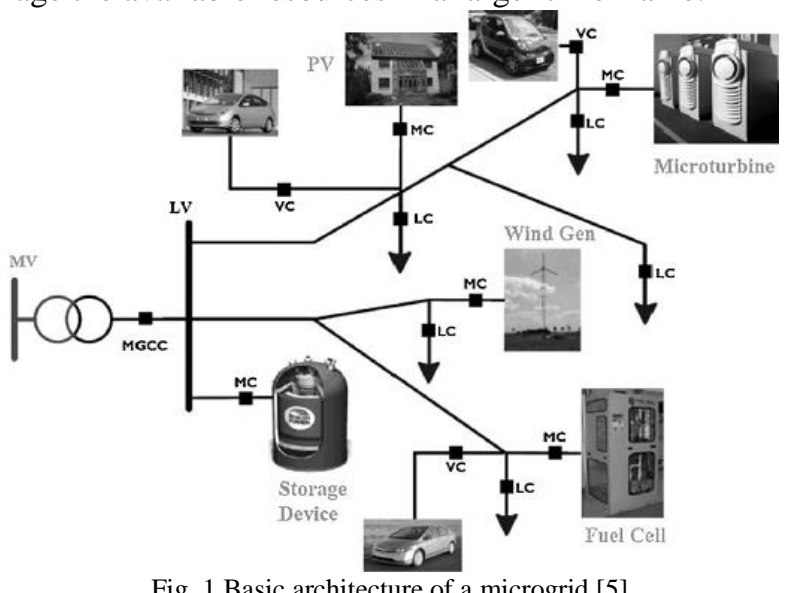

Fig. 1 Basic architecture of a microgrid [5].

\section{A. MG control functionalities}

When the MG disconnects from the upstream MV grid, it is necessary to establish the voltage reference in magnitude and frequency. Therefore, the MG requires specific primary and secondary frequency control strategies, in order to maintain power balance between generation and load and restore frequency to adequate values [12]. The implementation of frequency regulation strategies have to consider the MG resources controllability, namely: the storage device response, EV charging controllability, controllable MS response and load shedding schemes [7], [12], [13].

In the absence of synchronous machines, the MG requires storage units with the capability of providing fast power balance (i.e. flywheels, ultracapacitors, etc.). The storage unit coupling inverter is controlled as a voltage source inverter with external droop control loops, defining the MG voltage and frequency references as a function of the grid operating conditions, as in (1)

$$
\begin{aligned}
& \omega=\omega_{0}-k_{P} \times P \\
& V=V_{0}-k_{Q} \times Q
\end{aligned}
$$

where $P$ and $Q$ are the inverter active and reactive power outputs, $k_{P}$ and $k_{Q}$ are the droop slopes (positive quantities) and $\omega_{0}$ and $V_{0}$ are the idle values of the angular frequency and voltage (values of the inverter angular frequency and terminal voltage at no load conditions).

As shown in (1), whenever the frequency deviation differs from zero, storage devices would keep on injecting or absorbing active power, in order to ensure power balance. Therefore, the secondary frequency control ensures that after a disturbance the frequency returns to its nominal value [7], [12]. When participating in secondary control, controllable MS change their power output in order to compensate the power injected/absorbed by the storage unit(s). Both local and centralized secondary control strategies have already been proposed in the literature. Local secondary frequency control can be implemented at the controllable MS (i.e. Single-Shaft Micro-Turbines - SSMT and Solid Oxide Fuel Cells - SOFC) through proportional-integral controllers, as proposed in [7]. Centralized strategies at the MGCC level are used to define new active and reactive power set-points for the controllable
MS, taking into consideration the overall MG operating state. Secondary control may also include additional synchronization loops for a smooth reconnection to the main grid after islanding [12].

\section{$B$. Integrating $E V$ in $M G$ emergency operation}

The load controllability and distributed storage capacity provided by the EV connected to the LV network are additional resources that can be exploited for MG frequency regulation purposes. In [5], [6] the authors propose a P-f droop control strategy implemented at the EV coupling inverter, where the EV will change the power exchanged with the LV grid based on the MG frequency. As shown in Fig. 2, for frequencies around the nominal value (in this case, $50 \mathrm{~Hz}$ ) the EV will charge the battery at a pre-defined charging rate. If a disturbance occurs and the frequency drops below the deadband minimum frequency, the EV reduces its power consumption, thus reducing the load of the system. When the MG frequency overpasses the dead-band maximum frequency, the EV can also increase its power consumption. For large disturbances, causing the frequency to go below the zerocrossing frequency $\left(\mathrm{f}_{0}\right)$, the EV starts to inject power into the grid (Vehicle-to-Grid -V2G - functionality).

The participation of the EV in the MG frequency regulation mechanisms is limited to a pre-defined frequency range. When the MG frequency becomes out of this range, the vehicle will inject/absorb a fixed power, which can also be defined. The definition of the EV control parameters will depend on the EV charger characteristics and on the willingness of EV owners to participate in such services. These parameters may differ from grid to grid and can be changed remotely by the MGCC, in order to promote adequate coordination with the $\mathrm{MG}$ frequency regulation mechanisms (load shedding schemes, availability of energy storage devices and their state of charge).

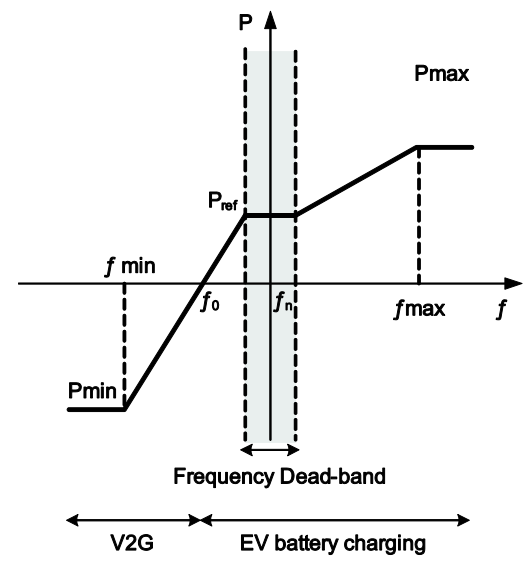

Fig. 2. Power-Frequency droop characteristic [6]

\section{Characterization of MG loads}

The MG can supply residential, commercial or small industrial customers. Residential and commercial loads are usually composed by resistive loads such as electrical heating and cooking appliances, lighting loads, electronic devices, thermostat-controlled loads and small induction motors. Thermostat controlled loads such as refrigerators, freezers, air conditioners and electric water and space heaters are 
continuously connected to the LV grid. This type of equipment is designed for switching between on and off states, in order to maintain the desired temperature within admissible range. However, due to their thermal storage, they can be disconnected during some minutes without harming consumers comfort.

The disconnection of other home appliances, such as washing machines, will delay for a few minutes their long operating cycles (in the order of 20 to 90 minutes), having low impact on consumers comfort. However, contrarily to thermostat controlled loads, the energy consumption patterns will vary more throughout the day.

In [9], the authors have grouped several home appliances, according to Table I, considering the maximum time they can be switched off without causing consumers discomfort. There is some uncertainty related to the effectiveness of the response of thermostat controlled loads to external on/off commands, since they can be operating in an off state when the off control signal is sent. However, this effect becomes attenuated when aggregating a larger number of devices [10], [14]. In this research, the uncertainty about demand response is mitigated, since it was assumed that a list of the responsive appliances which are in operation is available at the MGCC level, being periodically updated.

Table I. Characterization of Load Groups [9].

\begin{tabular}{c|cc}
\hline Load Group & Home Appliances & Maximum Time Off \\
\hline I & Electric space and water & $5 \mathrm{~min}$ \\
& heaters, Refrigerators, & \\
II & Freezers & $3.5 \mathrm{~min}$ \\
& Washing machines, & \\
III & Tumble Dryers & $2 \mathrm{~min}$ \\
IV & Cooking Appliances & $15 \mathrm{~s}$ \\
V & Electric in-line heaters & $4 \mathrm{~s}$ \\
\hline
\end{tabular}

\section{III.ADVANCED MG EMERGENCY FUNCTIONALITIES}

The MG control architecture previously described ensures continuous power balancing and frequency restoration to nominal values during islanding operating conditions. However, the effectiveness of the MG control strategies in the moments subsequent to the islanding will depend on several operating conditions, namely:

- MG storage capacity, which is essential to ensure primary frequency regulation.

- Controllable MS reserve capacity, in order to perform secondary frequency regulation.

- Non-controllable MS power production, which acts as a negative load in the system.

- MG flexible load, including the EV connected to the LV network.

- MG load in comparison to total generation.

In order to ensure the success of the MG autonomous operation, local resources have to be coordinated through adequate functionalities. The information sent by the local controllers to the MGCC can be used to characterize the MG operating conditions and identify a priori the most appropriate actions to take, if a certain disturbance affecting MG load and generation balance occurs. Updating the MG emergency operation strategies according to the MG actual operating state will allow a better coordination of the available resources, aiming to:

- Minimize the amount of load to be disconnected.

- Minimize the time during which loads are disconnected.

- Ensure that the MG has sufficient storage capacity to ensure primary frequency control following a given disturbance.

- Maintain frequency excursions within admissible limits.

Taking into consideration these objectives, an innovative online tool is proposed to be integrated at the MGCC level, which is responsible for periodically characterizing the $\mathrm{MG}$ operating state and evaluate the $\mathrm{MG}$ resilience in the event of a disturbance affecting MG load and generation balance. A set of critical disturbances can be identified in order to be analyzed $a$ priori by the proposed tool (such as unplanned islanding, changes in generation and load during islanding conditions, etc.). From the evaluation to be performed, the MGCC will define a set of actions to be parameterized at the local control level (load disconnection in LC, EV disconnection or droop parameterization at VC). Being an online tool running periodically, it defines a priori actions to be taken by the local controllers, which will be effectively activated if an emergency situation occurs. The activation of the local control actions can be deployed based on the local frequency measurement (at LC and VC), which can be compared to a frequency threshold defined by the online tool. The activation/parameterization of the controls that are going to locally decide about the load disconnection is performed previously to the disturbances, through short messages sent from the MGCC to the LC / VC on a periodic basis.

\section{A. Analyzing the $M G$ resilience}

According to the MG operational strategies previously described, frequency is the main indicator of the unbalance between generation and load. When a disturbance occurs during islanding operation, storage devices (incorporating a voltage source inverter electronic interface) naturally respond to the system frequency, thus providing primary frequency regulation. However, if the MG storage devices lack the energy necessary to ensure power balance, during the time of response of the MG secondary control, the system will collapse. Therefore, the MG available energy storage capacity needs also to be considered in order to characterize $\mathrm{MG}$ resilience following a given disturbance.

The MG controllable MS will provide secondary frequency control, which consequently reduces the energy required from the storage units. For a specified time period, the energy $E$ injected/absorbed by the storage unit can be determined by (2)

$$
E=E_{\Delta P}-E_{M S}-E_{V E}-E_{R L}
$$

where $E_{\triangle P}$ is the energy resultant from the power unbalance after the MG disturbance, $E_{M S}$ is the energy provided by the MS (both controllable and non-controllable) and $E_{V E}$ is the energy resultant from the response of the EV to the frequency deviation. $E_{R L}$ results from: (1) the energy not supplied to responsive loads, which were disconnected due to the emergency state of the MG or (2) the energy to be supplied to 
dump loads, in case the disturbance leads to an excess of generation regarding the MG load.

In addition to the MG frequency deviation in case of a disturbance, equation (2) enables the evaluation of the energy required to ensure secure islanded operation. The energy injected/absorbed by the MG storage unit will depend on the response of the controllable MS, typically SSMT and SOFC. Since these units present a non-linear power response, the dynamic models described in [13] were adopted instead of simplified linear models, which could lead to significant errors. As an illustrative example, Fig. 3 depicts the response of a SSMT to different power steps, leading to different responses in time.

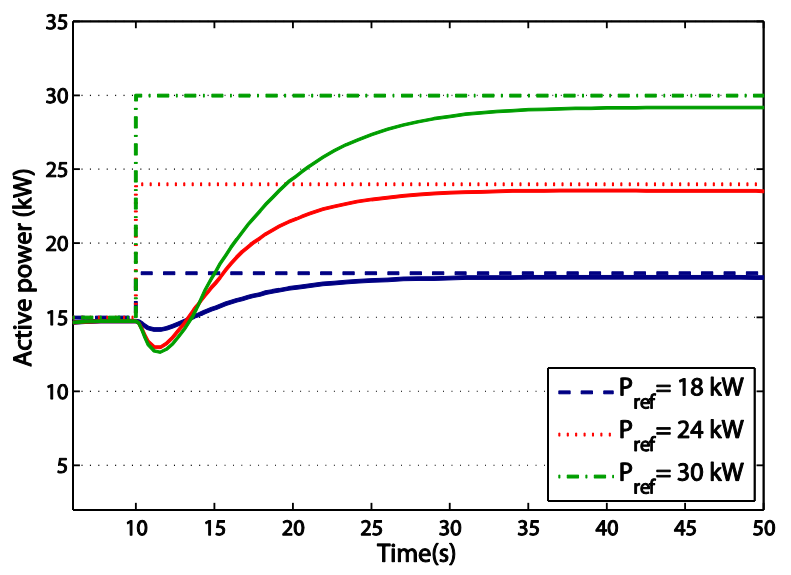

Fig. 3. SSMT step response (solid lines) to distinct reference powers (dashed lines).

However, some degree of simplification is required since the model should run periodically at the MGCC level and it cannot create a large computation load. The proposed simplifications concern the representation of the grid (which is three-phase, four wire), as well as the converters control loops since they act in a very fast way in comparison with the full MG dynamics. Under these conditions, Fig. 4 shows the simplified MG model, which consists on a single bus equivalent model. It allows studying the MG frequency response and determines the energy injected/absorbed by the storage unit.

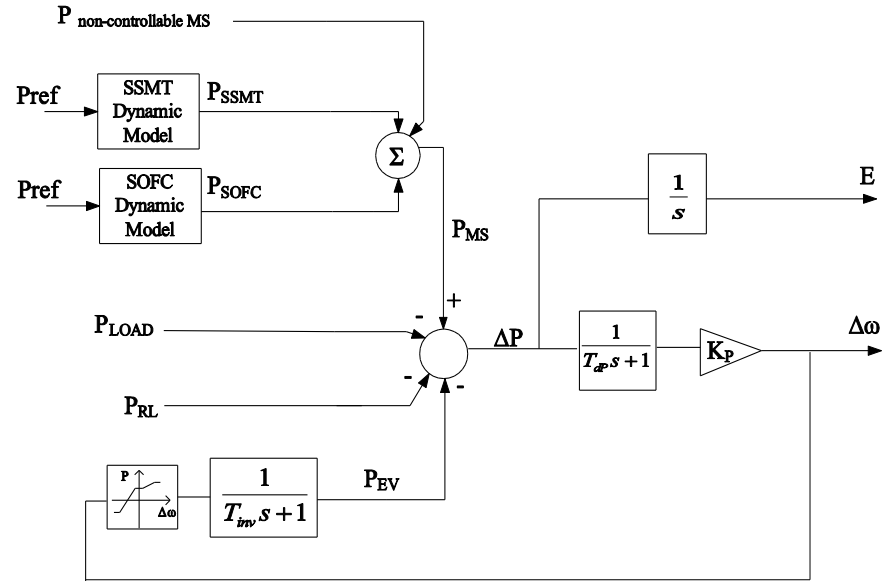

Fig. 4. MG simplified dynamic model to run at the MGCC.
The storage unit and EV are represented by their external P-f control loop. $T_{d P}$ is the delay related to the response of voltage source inverters and $T_{i n v}$ is the delay of the EV grid coupling inverter [13]. Loads and non-controllable MS are represented as constant active power.

\section{B. Managing the MG energy balance following islanding}

The tool proposed in this paper will be integrated in the MGCC and will run online for both interconnected and islanding modes of operation. When operating interconnected to the MV grid, the MG frequency is determined by the upstream network. Possible generation or load disturbances will only impact the power exchange between the MG and the MV network. Therefore, the main concern is related to the occurrence of unplanned islanding. However, during $\mathrm{MG}$ islanding operation, sudden changes on the MG load or power generation will cause frequency disturbances, which may compromise the stability of the system, either by excessive frequency deviation or by the unavailability of energy for load and generation balancing.

The algorithm proposed for defining the MG emergency operation strategy is represented in Fig. 5. The first step of the algorithm consists in identifying the MG operating mode. Then, based on the information sent by the local controllers, the MGCC will evaluate the available resources, namely: storage devices' State of Charge (SOC), controllable MS reserve (R), EV and load controllable power. If the $\mathrm{MG}$ is operating interconnected to the main grid, the occurrence of an unplanned islanding will cause an active power unbalance $\left(P_{\text {dist }}\right)$ equal to the power exchanged with the MG. If the MG is operating islanded, the power unbalance will result from the changes in loads or generation. It is important to notice that planned islanding events are not a key concern, since adequate control action can be taken in order to balance the MG load and generation prior to islanding, thus minimizing the associated transient phenomena.

In order to effectively study the MG frequency behavior and the energy balance problem for a given period of time, the model represented in Fig. 4 needs to consider a possible dispatch strategy for the controllable MS. The active power set-points to be sent to the MS controllers associated to controllable MS is assumed to represent a centralized secondary control mechanisms, which assigns power to controllable MS $i$ according to its reserve capacity, as defined in (3),

$$
\left\{\begin{array}{l}
d P_{i}=P_{d i s t} \frac{R_{i}}{R} \\
\sum_{i=1}^{n} d P i=P_{d i s t}
\end{array}\right.
$$

where $d P_{i}$ is the emergency active power step of unit $i, P_{\text {dist }}$ is the active power unbalance after islanding and $R_{\mathrm{i}}$ is the reserve capacity of unit $i$.

For a given disturbance, if it is identified that the MG does not have enough reserve capacity, it is necessary to exploit emergency responsive loads. The LC will be parameterized in order to disconnect some part of the MG load, ensuring power balance. The MG load to be disconnected is determined as in (4), 


$$
\Delta P=R-P_{\text {dist }}
$$

where $\Delta P$ is the amount of load to be disconnected, considering the available generation reserve $R$. This portion of the MG load will only be partially or totally reconnected after the re-synchronization with the main grid, or when sufficient reserve is achieved during the $\mathrm{MG}$ islanding stage.

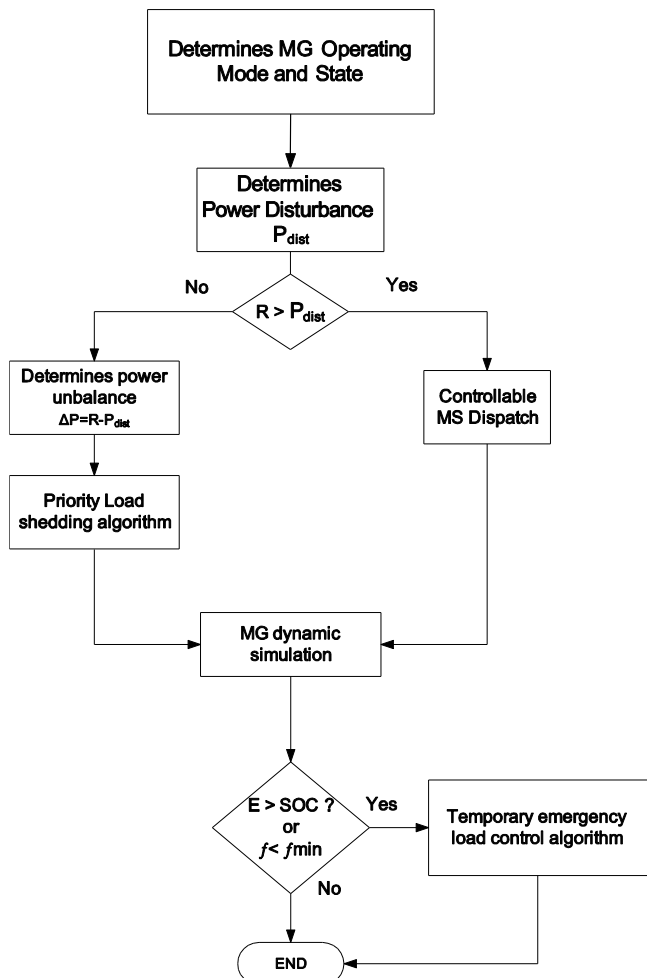

Fig. 5. Algorithm for defining the MG emergency operation strategy.

The initial responsive load disconnection strategy and the generation emergency dispatch are used as inputs of the MG dynamic model, in order to evaluate the energy balance within the $\mathrm{MG}$ for a given period and the expected frequency deviation in the event of a disturbance. As outputs, the model provides the total energy injected by the storage units and the MG frequency response (model from Fig. 4). Based on these values, the algorithm then verifies if the MG storage units have sufficient capacity to ensure power balance and if the minimum frequency does not violate the admissible frequency limits $\left(f_{\min }\right)$. If these conditions are violated, it is possible to follow an iterative procedure in order to determine an additional amount of load to be disconnected and compensate the slow response of MS to power control signals. The frequency threshold for its activation can also be identified, based on the frequency response obtained from the MG simplified model.

\section{Emergency Demand Response Strategy}

MG load control is required in two distinct situations:

- MG with insufficient generation reserve: in this case, the load will have to be curtailed until the system reconnects to the upstream network or the generation increases.

- MG with sufficient reserve: although the MG has sufficient reserve, it may happen that for a certain disturbance the storage SOC or minimum frequency conditions surpass admissible limits. The implementation of a temporary load curtailment will help improve the MG frequency regulation capacity and reduce the solicitation from the storage unit(s).

As previously stated, key objectives of the demand response strategy is to minimize the consumer's discomfort, thus involving the minimization of the disconnected load and time of disconnection. In order to avoid the disconnection of the consumer's load, the LC should be equipped with a dedicated functionality to manage controllable loads. As discussed in section II, some loads can only be disconnected during a limited time, like refrigerators and freezers. On the other hand, loads such as pool pumps may be considered non-priority loads when compared to other home appliances.

In order to minimize the consumer's discomfort, this paper proposes a selective load control scheme, where the consumer may prioritize the availability of its home appliances to participate in the MG load control scheme, as indicated in Table II. The first elements of the list are considered nonpriority loads and will be the first candidates to participate in the MG demand response scheme.

Table II. Load Group Characterization

\begin{tabular}{c|l}
\hline Load Group & \multicolumn{1}{c}{ Appliance } \\
\hline I & Pool pumps \\
II & Space and water heaters, refrigerators \\
III & Washing machines \\
IV & Electric cookers \\
\hline
\end{tabular}

The LC will monitor the power consumption of each load group and send the information to the MGCC, where it will be processed and aggregated per load category. Based on the load priority list and on the power unbalance, the load shedding algorithm defines what groups of loads should be disconnected, as shown in Fig. 6.

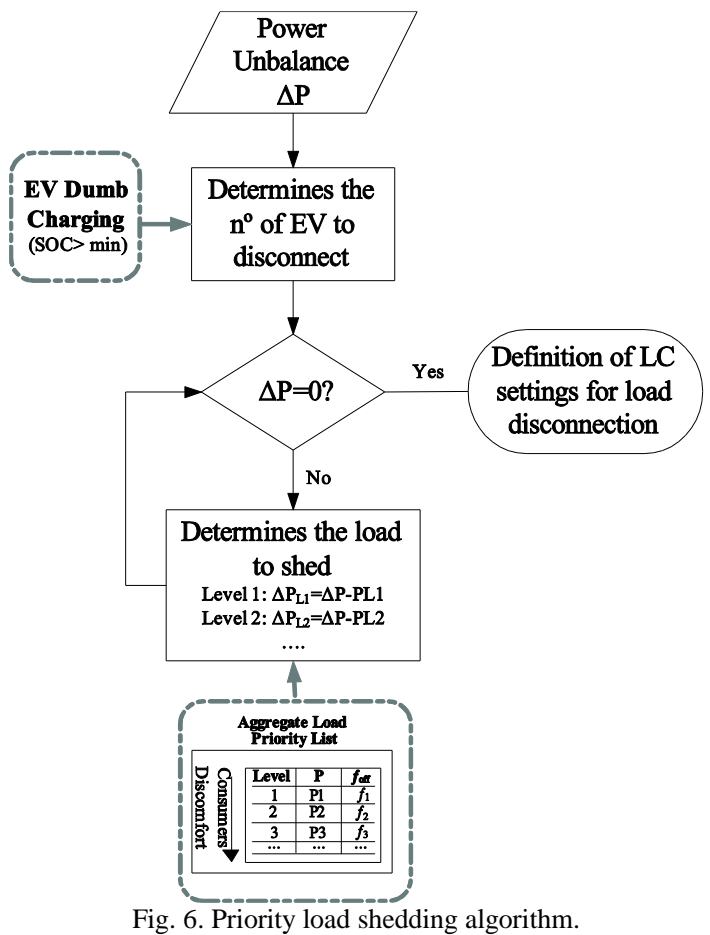


The MGCC will also use the information sent by the EV controllers (VC), differentiating between dumb charging and smart charging EV adherents. When adopting a dumb charging mode, the EV cannot be controlled through P-f droop strategy, behaving as a conventional (constant) load. In this case, the algorithm will disconnect all the dumb charging EV with a SOC higher than $80 \%$. If disconnecting the dumb charging $\mathrm{EV}$ is not sufficient to fulfill $\Delta P$, the load priority list is taken into consideration.

The on/off control of loads is based on the LC frequency measurement. In order to avoid over-shedding, the algorithm in Fig. 5 defines a disconnection frequency to the selected loads. This frequency set-point will depend on the frequency deviation estimated through the simplified dynamic model (see Fig. 4). When there is available reserve capacity, load reconnection takes place when the frequency recovers to a value close to the nominal one (for example, at least $49.9 \mathrm{~Hz}$ ). In order to avoid simultaneous load reconnections, a random time delay is considered for load reconnection after the moment frequency effectively reaches the pre-defined value.

\section{IV.MICROGRID TEST SYSTEM}

A typical urban LV distribution network (represented in Fig. 7) was used, in order to demonstrate the effectiveness of the proposed control strategies. The LV network has a nominal voltage of $400 \mathrm{~V}$ and supplies a total of 92 clients, from which 69 have a single-phase connection and the remaining 24 have a three-phase connection to the LV network. A 3.6 MJ flywheel capable of injecting $100 \mathrm{~kW}$ maximum power is assumed to be connected to the secondary side at the MV/LV substation. Three SSMT and a SOFC with $30 \mathrm{~kW}$ nominal power are connected in different network nodes. Four non-controllable MS, namely three single-phase photovoltaic panels and a single-phase micro wind turbine were also considered. The MG generation and load conditions are summarized in Table III.

The EV connected to the LV network are assumed to be connected to the MG through single-phase chargers, providing slow charging modes (taking about 8-12 $\mathrm{h}$ to reach full autonomy) [5]. At the nominal frequency, the power absorbed was considered $75 \%$ of the nominal power (about $3 \mathrm{~kW}$ ). The EV frequency-droop parameters are presented in Table IV.

Regarding the MG controllable loads, 3 load groups were considered for the load priority list and are defined in Table V. A minimum admissible frequency of $49.5 \mathrm{~Hz}$ and a minimum storage unit SOC of $50 \%$ were assumed for the operation during islanding conditions. The frequency for which the loads will be disconnected will be determined according to the results obtained from the MG simplified model (Fig. 4). If a temporary load curtailment is scheduled, the loads will be reconnected when the frequency returns to values higher than $49.9 \mathrm{~Hz}$ and if the MG has reserve capacity for additional load reconnection.

In order to validate the MG simplified model previously presented in Section III, a full MG model was also considered. The full MG model was developed in the Matlab/Simulink environment through the use of the SymPowerSystems toolbox and user-defined models, according to [5]-[7]. Detailed description of the dynamic models of MS, storage devices and EV chargers adopted can be found in [7], [13].

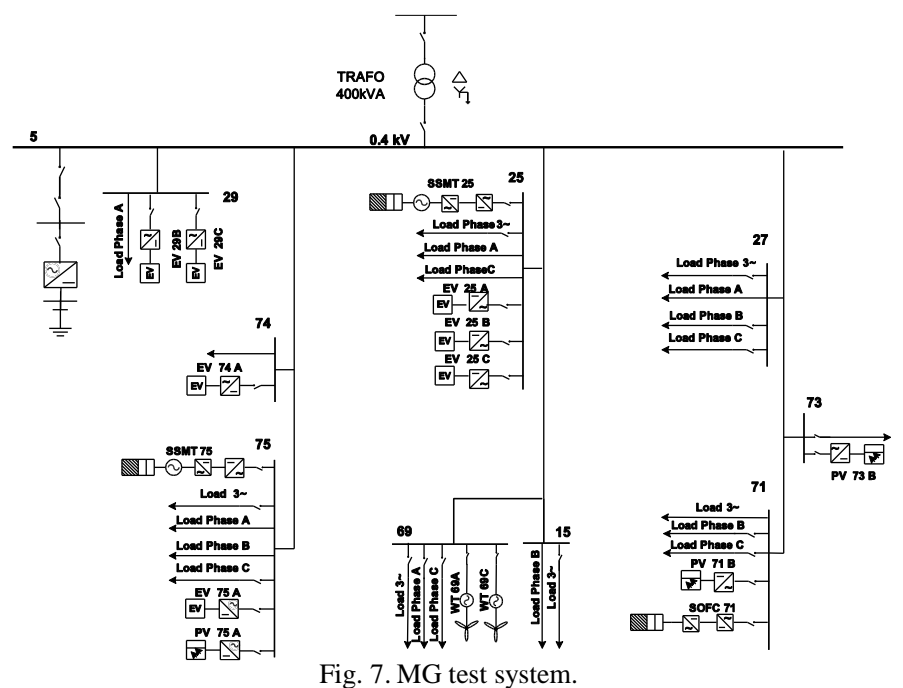

TABLE III. MG GENERATION CAPACITY AND LOAD

\begin{tabular}{c|llllll}
\multicolumn{1}{c}{} & \multicolumn{5}{c}{ Type of Connection } \\
\cline { 2 - 6 } \multicolumn{1}{c}{ 3 } & A & B & C & Total \\
\hline Load $(\mathbf{k W})$ & 42 & 20 & 25 & 33 & 120 \\
MS (kW) & 120 & 8 & 16 & 12 & 156 \\
EV $(\mathbf{k W})$ & - & 15 & 9 & 12 & 36 \\
\hline
\end{tabular}

TABLE IV. EV FREQUENCY- DROOP PARAMETERS

\begin{tabular}{ll}
\hline Frequency-Droop Parameters & \multicolumn{1}{c}{ Values } \\
\hline Nominal Frequency $(\mathrm{Hz})$ & 50 \\
Zero-Crossing Frequency $(\mathrm{Hz})$ & 49.5 \\
Maximum Frequency $(\mathrm{Hz})$ & 51 \\
Minimum Frequency $(\mathrm{Hz})$ & 49 \\
Frequency Dead-band $(\mathrm{Hz})$ & 0.2 \\
\hline
\end{tabular}

Table V. Controllable Load AVAilability

\begin{tabular}{cl}
\hline $\begin{array}{c}\text { Load } \\
\text { Group }\end{array}$ & $\mathrm{P}(\mathrm{kW})$ \\
\hline I & 30,2 \\
II & 14,3 \\
III & 18,3 \\
\hline
\end{tabular}

\section{V.SIMULATION RESULTS AND DISCUSSION}

In order to demonstrate the effectiveness of the emergency control of responsive loads, two scenarios were considered: in the first scenario the MG lacks sufficient reserve capacity to supply the MG loads after the islanding; in the second scenario the MG has sufficient reserve capacity to supply the MG loads. In both scenarios the disturbance under analysis is an unplanned islanding, which does not allow to balance $\mathrm{MG}$ load and generation prior to the islanding. In both cases the MG was importing power from the MV network before the islanding. For the second scenario, a sudden loss of renewable generation during the initial phase of the islanding condition was also considered.

\section{A. Scenario 1-MG with reserve capacity shortage}

In this scenario the SOFC is disconnected from the MG and the SSMT are producing approximately half of their nominal power. Consequently, the MG is importing about $75 \mathrm{~kW}$ of active power prior to the islanding. Based on this information, the control strategy described in section III defines an 
emergency dispatch for the controllable MS and determines the amount of responsive loads to shed. Since the amount of power imported from the main grid is higher than the MG reserve capacity, the MS emergency dispatch fixes the MS reference power at their nominal values and defines $36.3 \mathrm{~kW}$ of load shedding.

Regarding the participation of EV in the emergency load control strategy, two cases were considered: a first case considering that $100 \%$ of the EV connected to the LV network are controlled through P-f droop and a second scenario where $50 \%$ of the EV connected to $\mathrm{LV}$ network adopt a dumb charging mode (fixed charging rate).

\section{1) $100 \%$ of the EV connected to the MG participate in the primary frequency regulation}

At the beginning of the simulation, the MG is connected to the MV network, being disconnected at $t=10 \mathrm{~s}$. Since all the $\mathrm{EV}$ are participating in the MG primary frequency regulation, the load priority algorithm determines the disconnection of all the loads from group I and $42.3 \%$ of the loads from group II. The loads will be disconnected when the MG frequency decreases below $49.7 \mathrm{~Hz}$. This value was chosen as it is close to the minimum value admissible for the MG operation. As it is shown in Fig. 8, if no load is disconnected, a higher frequency deviation will occur and the MG storage unit and the EV will continue to inject power to compensate the unbalance.

For model validation purposes, Fig. 8 also compares the MG frequency response using the MG simplified model and the full MG model. In both models, the MG frequency does not reach the nominal frequency, stabilizing at $49.96 \mathrm{~Hz}$. This frequency deviation is caused by the response of the SSMT, which do not reach their nominal power, due to internal losses in the turbines. Fig. 9 presents the response of the storage unit obtained through both simulation models. Since the $\mathrm{MG}$ frequency remains at $49.96 \mathrm{~Hz}$, the storage unit will keep injecting a residual amount of power $(3.63 \mathrm{~kW})$.

The results shown in Fig. 8 and Fig. 9, demonstrate the accuracy of the MG balance model in comparison to the full MG model, when analyzing the MG frequency stability and power balance. Following the validation of the simplified MG model, it is used to derive the results presented in the next subsections.

\section{2) $50 \%$ of EV participating in the $M G$ primary frequency regulation}

In this case, it was considered that the EV adopting a dumb charging strategy had a battery SOC higher than $80 \%$. Therefore, according to the algorithm in Fig. 6, these EV will be disconnected, representing an aggregated load of $15.8 \mathrm{~kW}$. However, as shown in Fig. 10, if only the EV were disconnected the MG frequency wouldn't recover to the nominal value, stabilizing at $49.82 \mathrm{~Hz}$. Therefore, an additional curtailment of $20.5 \mathrm{~kW}$ of the loads from group I was considered. Comparing case 1 and 2, the reduction of the number of $\mathrm{EV}$ participating in the $\mathrm{MG}$ primary frequency regulation caused a higher frequency deviation.

Fig. 11 shows, the storage unit available energy after the islanding. For case 1 and 2 (considering $100 \%$ and $50 \%$ of EV controlled through P-f droop) the load curtailment strategies minimized the energy injected by the storage unit, maintaining its state of charge at approximately $80 \%$.
Otherwise, the MG security during islanding would be compromised.

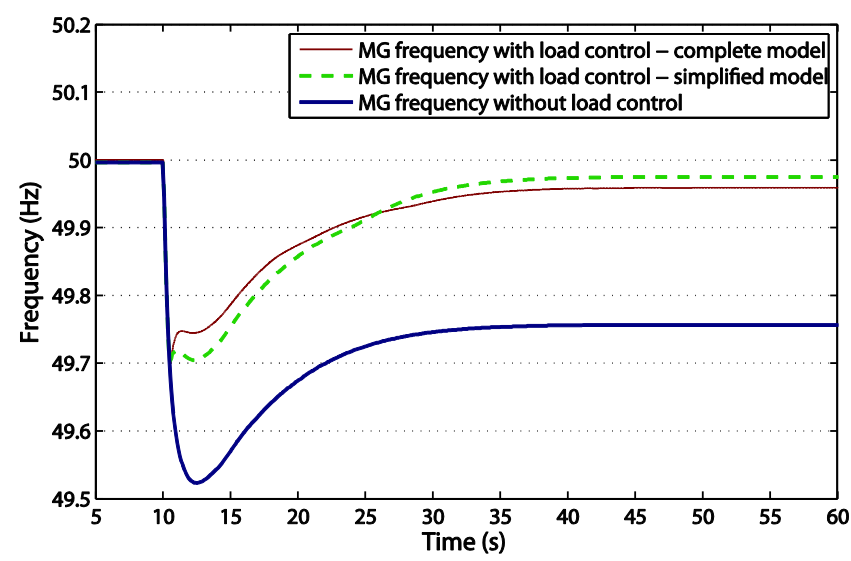

Fig. 8. MG frequency after the islanding and with $100 \%$ of EV participating in the MG primary frequency regulation: without load control, with load control for the complete model and simplified model.

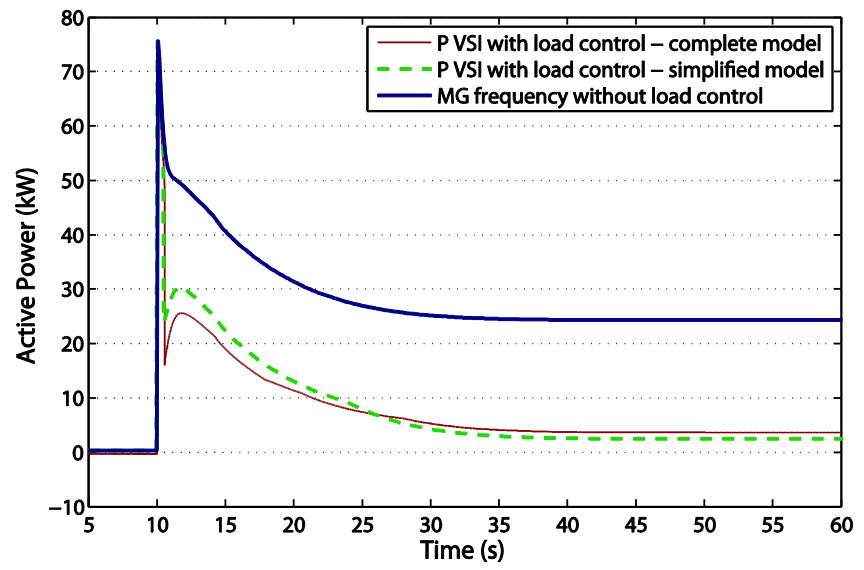

Fig. 9. Active power injected by the storage unit during islanded operation: without load control, and with load control for the complete model and simplified model.

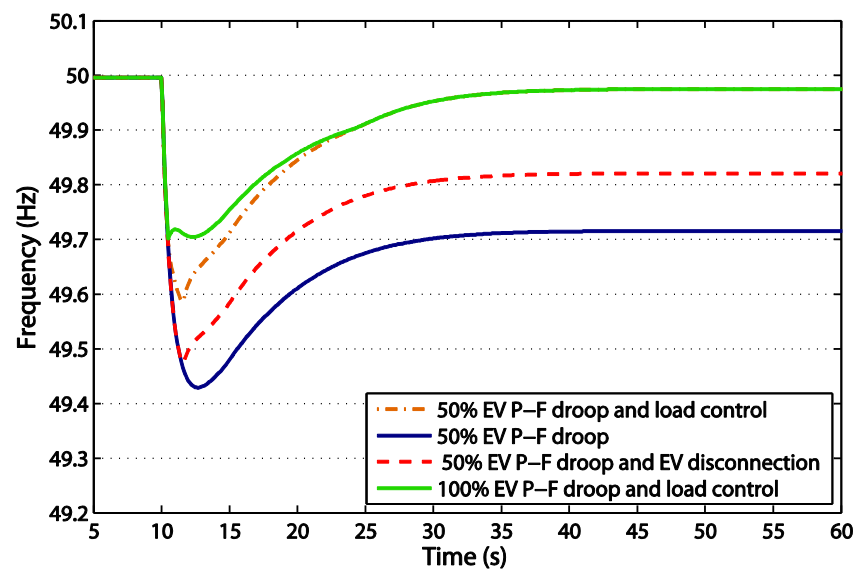

Fig. 10. MG frequency for scenario 1 considering the emergency load control strategy and participation of EV. 


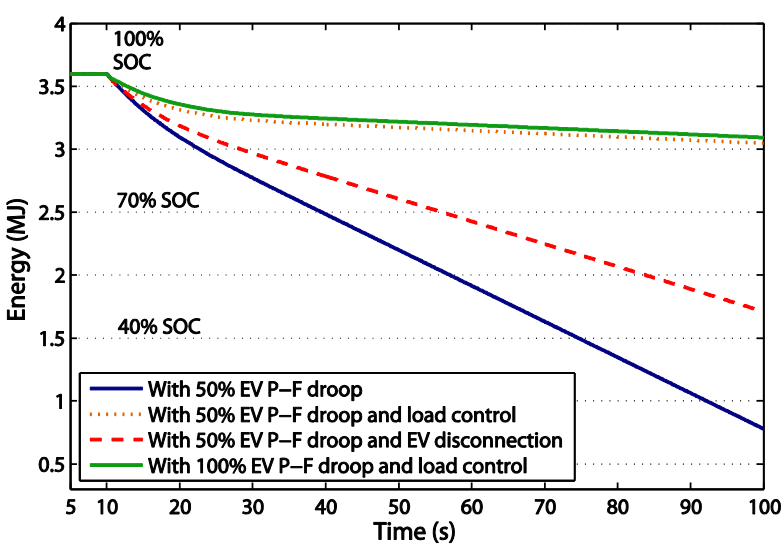

Fig. 11. Storage unit available energy after the islanding for Scenario 1, considering the emergency load control strategy and participation of EV.

\section{B. Scenario 2-MG with sufficient reserve capacity to supply} the MG loads

In this scenario the MG was importing $60 \mathrm{~kW}$ to supply a total load of $80 \mathrm{~kW}$. The SOFC in node 71 is connected to the MG, thus increasing the available reserve capacity. However, the initial power output of the SSMT and SOFC was low, totalizing $21 \mathrm{~kW}$. The initial storage unit state of charge was assumed to be $80 \%$.

Two distinct disturbances were simulated, namely: an unplanned islanding and a sudden renewable power shortage in the initial phase of the MG autonomous operation. If an unplanned islanding occurs, the MG has sufficient reserve capacity to supply the loads. However, it is necessary to ensure that the MG has sufficient storage capacity to comply with the storage unit criterion: to be always higher than $50 \%$.

As shown in Fig.12 a) and Fig. 13, if the MG only depends on its storage unit and controllable MS to provide frequency regulation, a $0.8 \mathrm{~Hz}$ frequency deviation will be experienced, causing more than $30 \%$ drop on the storage unit state of charge.

As expected, frequency deviation for the MG unplanned islanding overpasses the $49.5 \mathrm{~Hz}$ limit. Therefore, the primary frequency regulation needs to be complemented with additional mechanisms (EV control and load disconnection). Three different cases were then simulated: a first case considering only responsive loads, a second case considering only the participation of EV through the P-f droop characteristic and third case considering that only $50 \%$ of EV are available for participating as well as loads. Fig. 12 a) and b) compare the MG frequency response obtained for the different cases.

\section{1) Load control strategy for the MG islanding transient}

During the interconnected operation, the algorithm integrated in the MGCC defined for case 1 (only responsive loads) a temporary load shedding of $24 \mathrm{~kW}$ of the loads from group I, in order to reduce the initial frequency deviation. As shown in Fig. 12 a), the temporary load shedding avoids surpassing the $49.5 \mathrm{~Hz}$ frequency limit. The loads were disconnected when the frequency reaches $49.7 \mathrm{~Hz}$, reducing the energy required from the storage unit to ensure power balance during the frequency restoration phase (secondary control). As shown in Fig. 13, the state of charge of the MG storage unit remained higher than 55\% after the islanding.
In case 2 (all the EV controlled through P-f droop), the minimum frequency remained close to $49.6 \mathrm{~Hz}$, as shown in Fig. 12 a). Therefore, the algorithm did not define any load curtailment. However, when only $50 \%$ of EV are controlled through P-f droop, a small amount of power (around $7.2 \mathrm{~kW}$ ) was temporarily disconnected at a frequency of $49.7 \mathrm{~Hz}$, in order to avoid frequency deviations lower than $49.5 \mathrm{~Hz}$. The disconnected load was reconnected in steps when the frequency reaches $49.9 \mathrm{~Hz}$, as it was previously discussed. In both cases, the MG storage unit state of charge remained above $50 \%$.

\section{2) Load Control strategy for the MG power disturbances} during islanded operation

After the islanding, the MG has a remaining reserve of 32 $\mathrm{kW}$. However, it is necessary to assure that the MG remains with a storage capacity higher than the defined criterion. As shown in Fig. 12 b) and Fig. 13, when the MG suffers a renewable power shortage equal to the remaining reserve, the storage state of charge will fall below $40 \%$, if no load is disconnected and the EV do not participate in the MG frequency control. The MG frequency remains higher than $49.5 \mathrm{~Hz}$, but in order to maintain a minimum SOC of $50 \%$, the algorithm defined a temporary curtailment of $10 \mathrm{~kW}$ for case 1 (only responsive loads). Similarly to the previous case, the loads are reconnected in small steps when the frequency recovers to $49.9 \mathrm{~Hz}$. In the other cases considering the participation of EV, no additional curtailment was required.

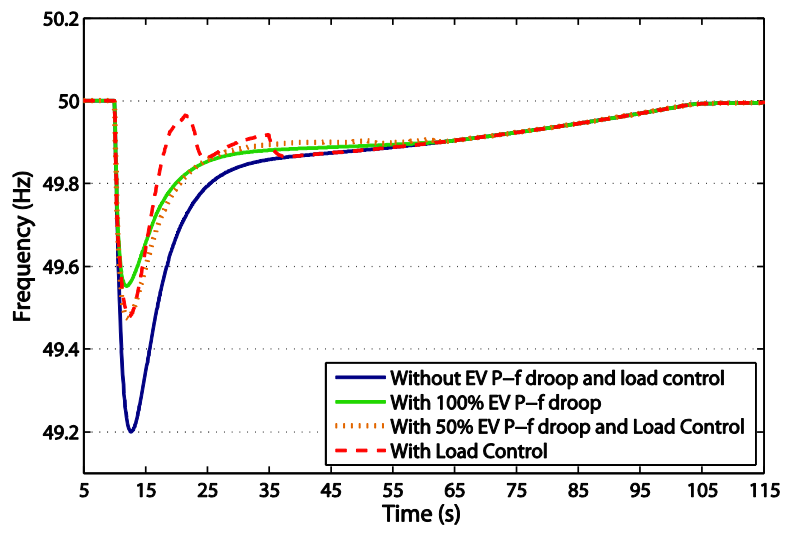

a)

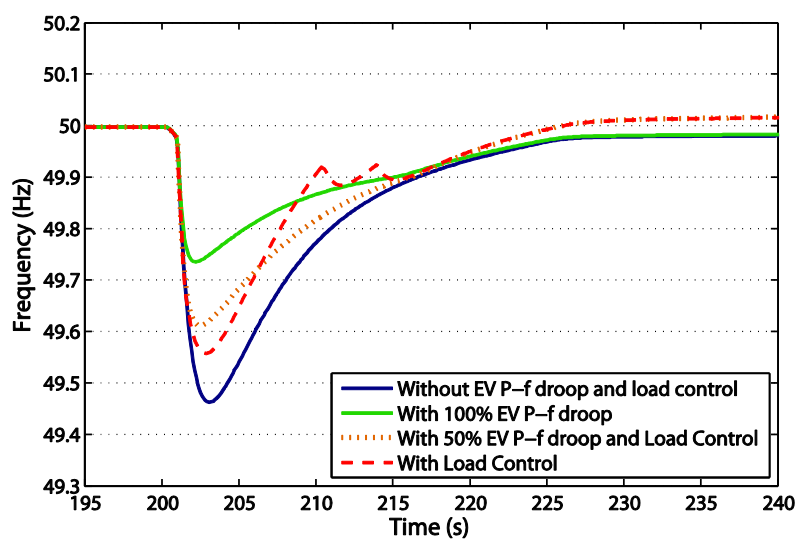

b)

Fig. 12. MG frequency for Scenario 2, considering the emergency load control strategy and participation of EV: a) after the islanding and b) after a sudden renewable power shortage. 


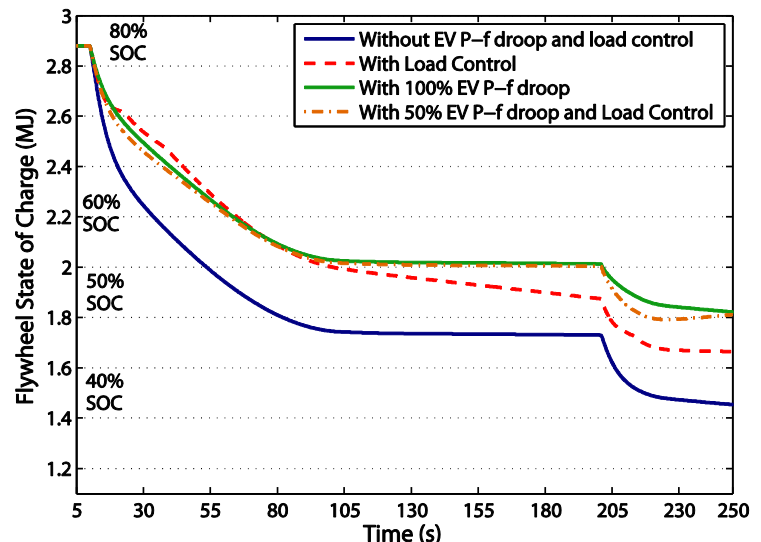

Fig. 13. Storage unit available energy for scenario 2 considering the emergency load control strategy and participation of EV.

In Fig. 12 a) and b) it is important to notice that the load control strategy has a strong impact in $\mathrm{MG}$ frequency response, in the initial moments subsequent to the transient. Afterwards, the MG frequency response has a strong dependence on the controllable MS dynamics (in particular, the response of SOFC which is typically slower than SSMT [7]). Also, the coordination of EV and load control strategies demonstrates great benefits in terms of the severity of the frequency disturbance and consequently of the storage solicitation. Further improvements on the reconnection of loads can be performed in order to minimize the transients.

\section{VI.CONCLUSIONS}

This paper presents the development of advanced MG load management functionalities, which are able to improve $\mathrm{MG}$ resilience following islanding, taking into account the $\mathrm{MG}$ limited energy storage capability and frequency response. The use of the proposed functionalities involves the exploitation of responsive loads as a complementary resource to MG primary frequency regulation. The process also includes the contribution provided by EV charging interfaces. The developed functionalities are of paramount importance for the MG online operation and management, aiming the seamless transition to islanding operation after the occurrence of contingencies in the upstream MV network, as well as a stable operation in the moments subsequent to islanding. From the results obtained for a $\mathrm{LV}$ test $\mathrm{MG}$, it is possible to conclude that the derived approach provided effective results. The illustrative cases evaluated through dynamic simulations, demonstrate the quality and the feasibility of the proposed tool when dealing with this problem.

The proposed approach is intended to support MG islanding operation during short periods of time (i.e. less than 1 hour). For larger time frames of operation in islanding conditions, complementary approaches need to be considered, involving forecasting of loads with different degrees of flexibility (including EV), as well as forecasts for renewable based microgeneration.

\section{REFERENCES}

[1] K. Moslehi, R. Kumar, "A Reliability Perspective of the Smart Grid," IEEE Transactions on Smart Grid, vol.1, no.1, pp. 57-64, June 2010.
[2] R.H. Lasseter, "Smart Distribution: Coupled Microgrids," Proceedings of the IEEE, vol.99, no.6, pp.1074-1082, June 2011.

[3] B. Lasseter, "Microgrids [distributed power generation]", IEEE Power Engineering Society Winter Meeting 2001, vol.1, no., pp.146-149 vol.1, 28 Jan-1 Feb 2001.

[4] Goran Strbac, "Demand side management: Benefits and challenges", Energy Policy, vol. 36, Issue 12, pp. 4419-4426, December 2008.

[5] J.A. Peças Lopes, Silvan A. Polenz, C.L. Moreira, Rachid Cherkaoui, "Identification of control and management strategies for LV unbalanced microgrids with plugged-in electric vehicles", Electric Power Systems Research, vol. 80, Issue 8, pp. 898-906, August 2010.

[6] J.A. Peças Lopes, F.J. Soares, P.M.R. Almeida, "Integration of Electric Vehicles in the Electric Power System," Proceedings of the IEEE, vol.99, no.1, pp.168-183, Jan. 2011.

[7] J.A. Peças Lopes, C.L. Moreira, A.G. Madureira, "Defining control strategies for MicroGrids islanded operation", IEEE Transactions on Power Systems, vol.21, no.2, pp. 916- 924, May 2006.

[8] F.C. Schweppe, R.D. Tabors, J.L. Kirtley, H.R. Outhred, F.H. Pickel, A.J. Cox, "Homeostatic Utility Control", IEEE Transactions on Power Apparatus and Systems, vol.PAS-99, no.3, pp.1151-1163, May 1980.

[9] K. Samarakoon, J. Ekanayake, N. Jenkins, "Investigation of Domestic Load Control to Provide Primary Frequency Response Using Smart Meters", IEEE Transactions on Smart Grid, vol.3, no.1, pp.282-292, March 2012.

[10] J.A. Short, D.G. Infield, L.L. Freris,"Stabilization of Grid Frequency Through Dynamic Demand Control", IEEE Transactions on Power Systems, vol.22, no.3, pp.1284-1293, Aug. 2007.

[11] S.A. Pourmousavi, M.H. Nehrir, "Real-Time Central Demand Response for Primary Frequency Regulation in Microgrids", IEEE Transactions on Smart Grid, vol.3, no.4, pp.1988-1996, Dec. 2012.

[12] J.M. Guerrero, J.C. Vasquez, J. Matas, L.G. de Vicuna, M. Castilla, "Hierarchical Control of Droop-Controlled AC and DC Microgrids-A General Approach Towards Standardization", IEEE Transactions on Industrial Electronics, vol.58, no.1, pp.158-172, Jan. 2011.

[13] A. Keyhani and M. Marwali,"Microgrids Operation and Control under Emergency Conditions", in Smart Power Grids 2011, Ed. Berlin, Springer-Verlag, 2012, ch. 12, pp. 351-399.

[14] A. Molina-García, F. Bouffard, D.S. Kirschen, "Decentralized DemandSide Contribution to Primary Frequency Control", IEEE Transactions on Power Systems, vol.26, no.1, pp.411-419, Feb. 2011.

\section{BIOGRAPHIES}

C. Gouveia graduated in Electrical Engineering in the Faculty of Engineering of the University of Porto - FEUP (2008), and is currently working towards her $\mathrm{PhD}$ in Power Systems, also from the University of Porto. She is a member of the INESC-TEC research team and her research is focused on the development of the MicroGrid concept integrating plugged-in electrical vehicles in the operation of the electric system.

J. Moreira graduated in Electrical Engineering in the Faculty of Engineering of the University of Porto - FEUP (2012). His main research interests are focused on microgrids dynamics and control, smart grids and smart metering.

C. L. Moreira received the B.S. and Ph.D. degrees in electrical engineering from the University of Porto, Porto, Portugal, in 2003 and 2008, respectively. Since 2010 he is the Smart Grid Area leader in the Power Systems Unit of the Institute for Systems and Computer Engineering of Porto (INESC Porto). He is also assistant professor in the Department of Electrical Engineering of the Faculty of Engineering of University of Porto since 2008. His main research interests are focused on microgrids dynamics and control, smart grids and smart metering.

J. A. Peças Lopes (M'80-SM'94) received the B.S. and Ph.D. degrees in electrical engineering from the University of Porto, Porto, Portugal, in 1981 and 1988, respectively. Since 2008, he has been a full Professor of the Department of Electrical and Computer Engineering, Faculty of Engineering, University of Porto. He is currently the Director of the Ph.D. Program on Sustainable Energy Systems of the Faculty of Engineering, University of Porto. He is also a Director of the Institute for Systems and Computer Engineering of Porto (INESC Porto). He was responsible for INESC Porto activities in several EU financed research projects, and he is author or coauthor of more than 200 papers, having supervised more than $25 \mathrm{Ph} . \mathrm{D}$. and M.S. thesis. 
(C) 2013 IEEE. Personal use of this material is permitted. Permission from IEEE must be obtained for all other uses, in any current or future media, including reprinting/republishing this material for advertising or promotional purposes, creat-ing new collective works, for resale or redistribution to servers or lists, or reuse of any copyrighted component of this work in other works. 